\title{
PV.1 Suppresses the Expression of FoxD5b during Neural Induction in Xenopus Embryos
}

\author{
Jaeho Yoon ${ }^{1,2}$, Jung-Ho Kim ${ }^{1,2}$, Sung Chan Kim ${ }^{1}$, Jae-Bong Park ${ }^{1}$, Jae-Yong Lee ${ }^{1}$, and Jaebong Kim ${ }^{1, *}$
}

\begin{abstract}
Suppression of bone morphogenetic protein (BMP) signaling induces neural induction in the ectoderm of developing embryos. BMP signaling inhibits neural induction via the expression of various neural suppressors. Previous research has demonstrated that the ectopic expression of dominant negative BMP receptors (DNBR) reduces the expression of target genes down-stream of BMP and leads to neural induction. Additionally, gain-of-function experiments have shown that BMP downstream target genes such as MSX1, GATA1b and Vent are involved in the suppression of neural induction. For example, the Vent1/2 genes are involved in the suppression of Geminin and Sox3 expression in the neural ectodermal region of embryos. In this paper, we investigated whether PV.1, a BMP downstream target gene, negatively regulates the expression of FoxD5b, which plays a role in maintaining a neural progenitor population. A promoter assay and a cyclohexamide experiment demonstrated that PV.1 negatively regulates FoxD5b expression.
\end{abstract}

\section{INTRODUCTION}

During the development of vertebrate embryos, bone morphogenetic protein (BMP) signaling negatively regulates neural induction (Dale and Jones, 1999; Hawley et al., 1995; Wilson and Hemmati-Brivanlou, 1995). Ectopic expression of dominant-negative BMP receptors (DNBR) or dissociation results in downregulation of BMP signaling and leads to neural induction in ectodermal explants (Xu et al., 1995). This phenomenon is known as default neurogenesis (Kuroda et al., 2005). BMPs are a subfamily of the transforming growth factor beta (TGF- $\beta$ ) superfamily and modulate various biological processes via activation of Smads, most notably Smads 1/5/8 (Dale and Wardle, 1999). During the neural induction of developing embryos, BMP signaling induces various target genes including MSX1, GATA $1 \mathrm{~b}$ and Vents, and these proteins act as neural suppres-

\footnotetext{
${ }^{1}$ Department of Biochemistry, Institute of Cell Differentiation and Aging, College of Medicine, Hallym University, Chuncheon 200-702, Korea, ${ }^{2}$ These authors contributed equally to this work.

*Correspondence: jbkim@ hallym.ac.kr

Received 17 October, 2013; revised 17 December, 2013; accepted 18 December, 2013; published online 6 March, 2014
}

Keywords: BMP, FoxD5b, neurogenesis, PV.1, Xenopus sors (Rogers et al., 2009; Shibata et al., 1998; Suzuki et al., 1997). Experimentally, over-expression of these BMP target genes inhibits neural gene expression and induces epidermal fates (Wilson and Hemmati-Brivanlou, 1995). Specifically, gainof-function studies have demonstrated that Vents participate in the inhibition of Geminin, Zic3 and Sox3 expression in the neuroectoderm (Rogers et al., 2008).

Previous research has shown that the Xvent family of proteins modulates dorso-ventral specification (Friedle and Knochel, 2002; Gawantka et al., 1995). BMP-4 signaling directly induces the expression of Xvent and suppresses neural and dorsal mesodermal fate (Gawantka et al., 1995). PV.1 is a transcription factor that belongs to the Xvent gene family. PV.1 contains a homeodomain and acts as repressor via its Cterminal domain (Ault et al., 1996; 1997; Hwang et al., 2002; 2003). Ectopic expressions in various gain-of-function studies have demonstrated that PV.1 suppresses dorsal mesodermal gene and neural gene expression, including the Chordin, Goosecoid and Zic3 genes, and induces the expression of ventral genes including wnt8 and XHox3 (Hwang et al., 2002; 2003).

FoxD5 is a forkhead/winged helix transcription factor that functions in a variety of differentiation processes (Jackson et al., 2010; Katoh and Katoh, 2004; Katoh et al., 2012; Pohl and Knochel, 2005). During early Xenopus embryo development, FoxD5 modulates undifferentiated neural ectodermal fate and suppresses differentiation towards the neural plate in developing vertebrate embryos (Fetka et al., 2000; Lee et al., 2009; Sullivan et al., 2001; Yan et al., 2009a; 2009b; Yu et al., 2002). The transcription of FoxD5 is regulated by various signaling pathways. For example, Sullivan et al. (2001) reported that FoxD5 expression is up-regulated by the over-expression of Siamois and Noggin but not by the over-expression of Wnt-8 or Chordin. In our previous research, we showed that the suppression of BMP signaling induces FoxD5b expression via AP1c-Jun/ FosB. Additionally, we found that the FoxD5a and $b$ promoters have two highly conserved 5 -flanking regions. The AP-1 binding site, which is involved in the FoxD5b expression induced by the suppression of BMP signaling, is located in this conserved region. Additionally, we have previously reported that increased BMP signaling negatively regulates FoxD5b expression and that the activity of the FoxD5b promoter is reduced by increased BMP signaling; however, the detailed mechanisms of this process have remained elusive.

Here, we confirmed that FoxD5b expression is negatively regulated by BMP signaling. The over-expression of PV.1 (one of the target genes of BMP) indicated that PV.1 directly sup- 
pressed FoxD5b expression. Additionally, a promoter assay revealed that PV.1 might regulate FoxD5b expression indirectly via Hox genes. These results suggest that BMP signaling suppressed FoxD5b expression via the induction of its target genes.

\section{MATERIALS AND METHODS}

\section{Embryo injection and explant culture}

Xenopus laevis embryos were obtained by artificial fertilization (Smith and Slack, 1983). Developmental stages were designated according to the scheme of Nieuwkoop and Faber (1967). RNA or DNA was injected into the animal pole of the embryos at the one-cell stage, as described in the figure legends. The animal caps were dissected from the injected embryos at stage 8 and cultured until stage 13 in $67 \%$ Leibovitz's L-15 medium (GIBCO/BRL) with BSA (1 mg/ml), $7 \mathrm{mM}$ Tris- $\mathrm{HCl}(\mathrm{pH} 7.5)$ and gentamicin $(50 \mu / \mathrm{ml})$. The cultured explants were incubated at $23^{\circ} \mathrm{C}$ before harvesting.

Whole mount in situ hybridization

Embryos were injected with mRNAs as indicated and subsequently processed for whole-mount in situ hybridization using standard methods with anti-sense probes for FoxD5b (Moore et al., 2004).

RNA isolation and reverse transcription-polymerase chain reaction ( $\mathrm{RT}$-PCR)

For qRT-PCR, total RNA was prepared using the TRIzol reagent (Tel-Test, Inc., USA), and cDNA was synthesized using the SuperScript pre-amplification system (Invitrogen). The PCR primers and cycling conditions are described in the Xenopus Molecular Marker Resource (University of Texas). Additional primers are described in Table 2. The PCR reactions were performed with SYBR Premix (Qiagen, USA) and a thermal cycler real-time system (Qiagen Rotor-Gene-Q, USA).

\section{In vitro transcription}

The PV.1 and DNBR mRNAs used for microinjection were produced by in vitro transcription. The cDNAs for PV.1 and DNBR were inserted into the pCS2 vector. The cDNAs were linearized and used for in vitro synthesis of capped mRNA using an in vitro transcription kit (Ambion) according to the manufacturer's instructions. The synthetic RNA was quantified with ethidium bromide staining and compared to a standard RNA.

\section{Luciferase assay}

The level of luciferase activity was measured using a luciferase assay system according to the manufacturer's instruction (Promega, USA). Five or six groups of animal caps (5 animal caps per group) were harvested and homogenized in $30 \mu \mathrm{l}$ of lysis buffer. A luminometer was used to measure $40 \mu$ of luciferase substrate and $10 \mu \mathrm{l}$ of whole cell lysate (Promega, USA). All experiments were repeated at least three times using independently derived sample sets.

Site-directed mutagenesis

Mutagenesis of -301_(m)Hox was performed using a SiteDirected Mutagenesis Kit (Intronbio, KR) according to the manufacturer's instructions. PCR primers were (upstream) 5 '-CA TCACATAGATGCGCGAGACTTAATTATTGG-3' and (downstream) 5'-CCAATAATTAAGTCTCGCGCATCTATGTGATG-3'.

Statistical analyses

All experiments were independently performed more than three times. The data are presented as the means \pm the SEs. T-tests were used to compare groups via the GraphPad Prizm program (GraphPad Software, USA).

\section{RESULTS}

Neural induction is negatively regulated by PV.1

PV.1 is a downstream target gene of BMP signaling (Ault et al., 1996; Lee et al., 2011) and has a ventralizing effect in Xenopus embryos (Ault et al., 1997; Hwang et al., 2003). To confirm the role of PV.1 in whole embryos, PV.1 RNAs were co-injected with beta-galactosidase into embryos in the one-cell stage. As shown in figure 1A, over-expression of PV.1 dose-dependently caused ventralization and abnormal head formation. These morphological changes suggest that PV.1 reduced dorsal mesodermal and neural gene expression. Therefore, to confirm that PV.1 inhibited the expression of neural genes, RT-PCR was performed on DNBR-injected ectodermal explants. Suppression of BMP signaling by DNBR induced expression of neural genes including Zic3 and FoxD5b without inducing dorsal mesoderm. However, over-expression of PV.1 decreased the expression of the neural genes Zic3 and FoxD5b (Fig. 1B). Additionally, we examined the temporal expression patterns of PV.1 and FoxD5b. PV.1 was highly expressed during the midblastula stage, and FoxD5b was also expressed from the early blastula to the mid-blastula stages (Fig. 1C). An in situ hybridization assay showed that the over-expression of PV.1 decreased FoxD5b expression in whole embryos (Fig. 1D).

Taken together, these data suggest that PV.1 regulates FoxD5b expression in blastula embryos.

\section{PV.1 directly suppresses neural induction}

To investigate whether PV.1 directly or indirectly decreases FoxD5b expression, the transcriptional levels of FoxD5b were examined in cyclohexamide (CHX)-treated ectodermal explants by RT-PCR. As shown in Fig. 2A, suppression of BMP signaling induced FoxD5b expression, but co-injection of PV.1 significantly decreased the FoxD5b expression that was induced by DNBR in the control animal cap explants. Interestingly, coinjection of DNBR and PV.1 also led to same result in the cyclohexamide-treated animal cap explants. These data suggests that PV.1 directly reduced FoxD5b expression. Additionally, we also explored whether the expression of Zic3, one of the neural-specific genes, was regulated by PV.1. Our data showed that Zic3 was also directly suppressed by PV.1 (Fig. 2B). Taken together, these results suggest that PV.1 directly suppressed neural gene expression.

\section{PV.1 negatively regulates the promoter activity of FoxD5b}

To investigate how PV.1 negatively regulates FoxD5b expression, a promoter assay was performed with the $5^{\prime}$-flanking region of the FoxD5b promoter. Over-expression of PV.1 decreased the luciferase activity of the FoxD5b promoter (Fig. 3A). To identify the PV.1-response element, serial truncated FoxD5b promoters were analyzed. Our previous studies have shown that the AP-1 binding site, which acts as a positive regulatory element, is located in between -1336 and -1316 . Additionally, we found that two promoter regions of FoxD5a and FoxD5b were highly conserved as shown in Fig. 3B. Truncation of the $\mathrm{AP}-1$ binding site in the FoxD5b promoter decreased luciferase activity because the positive regulatory element was eliminated. Interestingly, over-expression of PV.1 decreased the luciferase activity of the serially truncated FoxD5b promoter, but the activity of the -186 construct was not reduced by PV.1. 
A
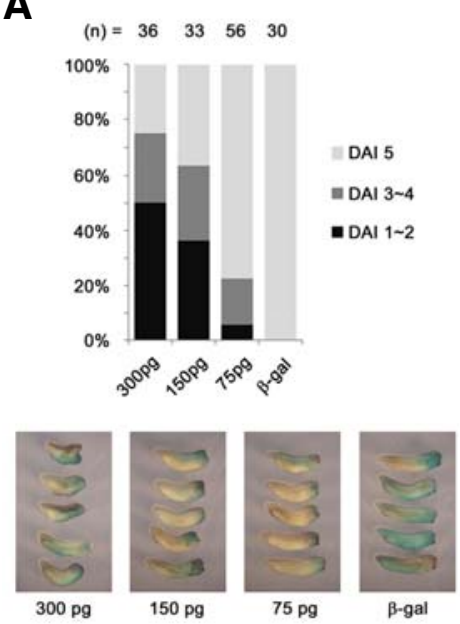

B

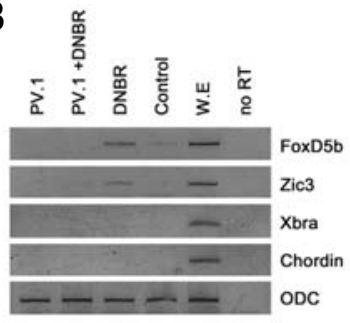

D

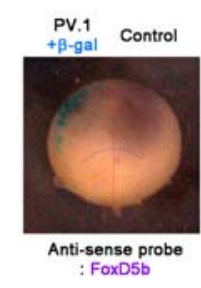

C

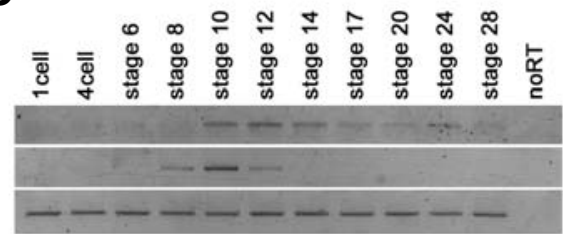

Fig. 1. PV.1 suppresses neural gene expression. (A) PV.1 RNAs of PV.1 was injected as indicated at the one-cell stage. Phenotypical changes were observed at stage 33. DAl means the dorso- anterior index. (B) PV.1 (500 $\mathrm{pg}$ ) was either injected alone or co-injected with DNBR $(1 \mathrm{ng})$ at the one-cell stage. Animal cap explants were dissected at stage 8 , incubated until stage 10 and then RT-PCR was performed for the analysis of relative gene expression. ODC, loading control; $\begin{array}{ll}\text { PV.1 } & \text { noRT, control reaction without } \\ \text { FoxD5b } & \text { reverse transcriptase; WE, Whole }\end{array}$ ODC embryo as a positive control; chordin, Dorsal mesodermal mar

ker; Xbra, mesodermal marker; FoxD5b and Zic3, early neural marker. (C) The temporal expression pattern of FoxD5b and PV.1 were analyzed using RT-PCR at various developmental stages as indicated. (D) PV.1 RNAs were injected into one side of each of the two cell embryos. These embryos were processed for whole mount in situ hybridization with anti-sense probe of FoxD5b at stage 10. The injected side of the embryos is indicated by Beta-gal staining. The blue dotted line indicates the dorsal lip.

A

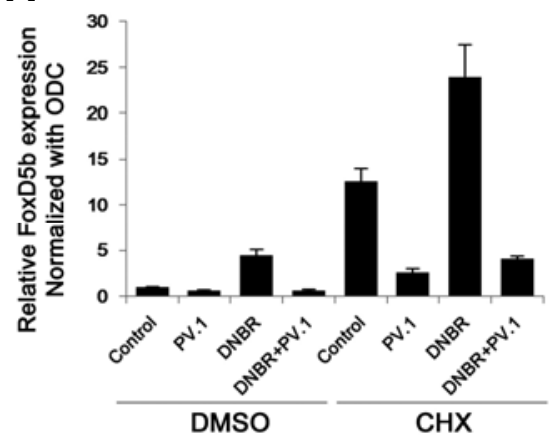

B

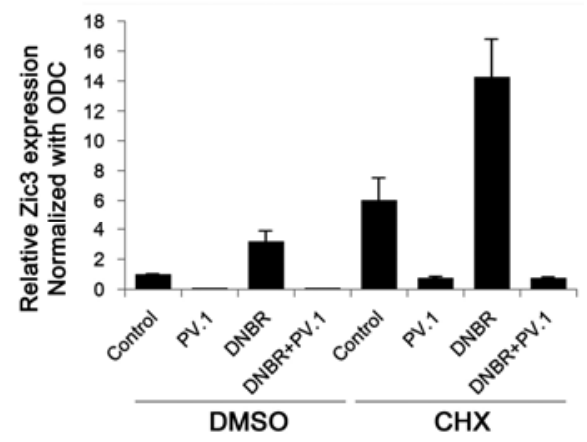

Fig. 2. PV.1 reduces FoxD5b and Zic3 expression. (A, B) Embryos were injected with RNAs (PV.1, $500 \mathrm{pg}$ and DNBR, $1 \mathrm{ng}$ ) at the one-cell stage as indicated. Animal explants were dissected at stage 8 and incubated in the animal cap media containing DMSO or cyclohexamide (CHX, $5 \mathrm{ng} / \mathrm{ml})$ until stage 10. RT-PCR was performed to analyze the relative FoxD5a and Zic3 expressions. Data are shown as the means \pm the S.D. of the values from at least 3 independent experiments. Differences were considered significant at $P<0.05$.
These results suggest that the PV.1 response element is located in between -301 and -186 , which is a conserved region in the FoxD5 and FoxD5b promoters.

\section{The indirect response element of the FoxD5b promoter is between -301 and -186 region}

We have previously shown that PV.1 negatively regulates FoxD5b expression and that the response element is located in the FoxD5b promoter between -301 and -186 . Thus, we next analyzed promoter sequences to identify the Xvent/PV.1 binding site (CAAATAA) (Taylor et al., 2006). However, the putative Vent/PV.1 binding site was not present, and chromate immunoprecipitation (ChIP) analysis also demonstrated that PV.1 could not bind in this region (data not shown). To confirm if the PV.1 response element is present in the region between -301 and -186 , the expression of the luciferase gene, which is encoded by the FoxD5b promoter, was measured using cyclohexamide-treated animal cap explants and RT-PCR. As shown in Fig. 4A, the expression level of luciferase mRNA, which is encoded by the FoxD5b promoter, was increased by the DNBR.
Co-injection of PV.1 with DNBR decreased luciferase expression in the DMSO-treated animal cap explants. However, overexpression of PV.1 did not change the expression of the luciferase gene in the cyclohexamide-treated animal cap explants. These results suggest that our FoxD5b promoter construct did not contain the direct PV.1 regulatory element; rather the indirect regulatory response element exists in this region. Interestingly, we found a putative Hox binding site in the region between -301 and -186 .

Hox genes are also BMP-target genes and are involved in ventral fate specification (Wacker et al., 2004). Thus, we generated the a -301_(m)Hox construct that contained a mutation in the Hox binding site. We compared the promoter activity of 301 and -301_(m)Hox in whole embryos as indicated in Fig. 4C. The decreased reporter activity of the wild type promoter by PV.1 was not found when $-301-(\mathrm{m}) \mathrm{Hox}$ was co-injected with PV.1. Thus, our data suggest that the putative Hox binding site mediates the suppression of FoxD5b expression due to the over-expression of PV.1. Additionally, the over-expression of PV.1 revealed that the expression of some Hox genes, include- 


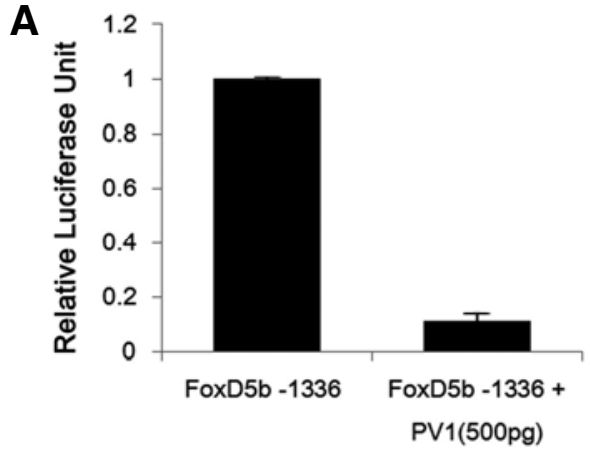

C

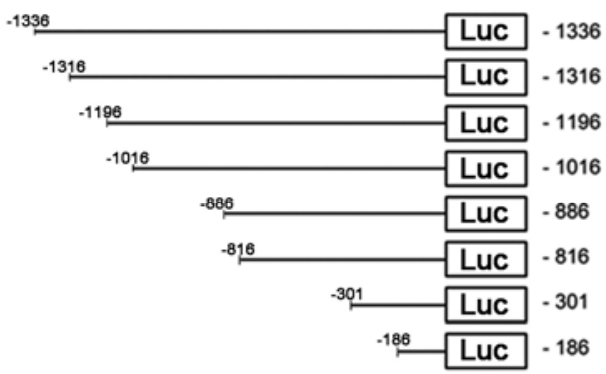

B

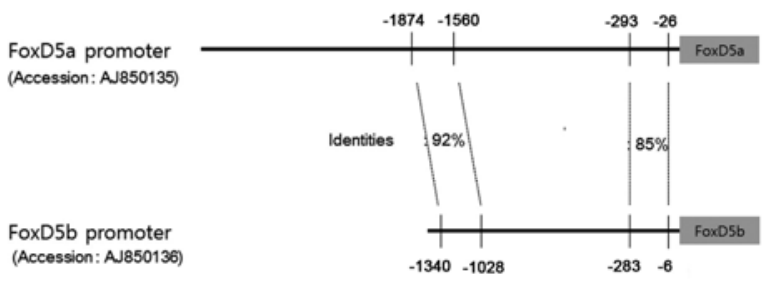

D

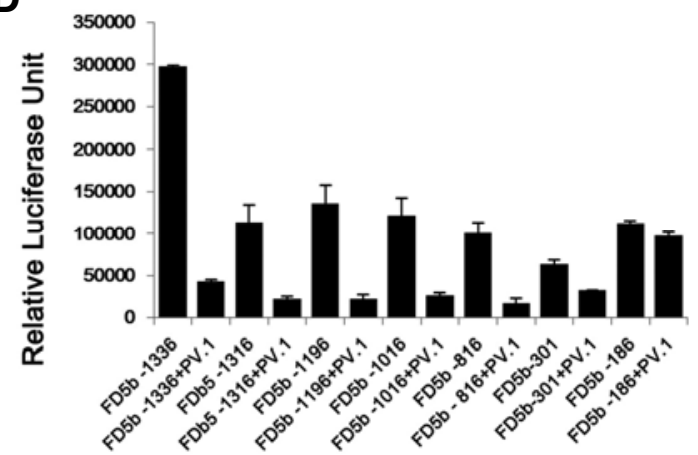

Fig. 3. PV.1 reduces the promoter activity of FoxD5b. (A) Embryos were co-injected with the -1336 construct (20 pg) and PV.1 (500 pg) at the one-cell stage and incubated until stage 10. Luciferase activity was measured as described in the "Materials and Methods". (B) The graph shows regions of high similarity based upon the NCBI-BLAST results. (C) Schematic representation of the serially truncated FoxD5b promoter constructs. (D) The embryos were injected with FoxD5b promoter alone or with PV.1 as indicated. Luciferase activities were measured at stage 10. The data are shown as the means \pm the S.D. of the values from at least three independent experiments. Differences were considered significant at $P<0.05$.

ing HoxB4 and HoxC6, were increased (Fig. 4D).

Taken together, we interpret our data to suggest that the suppression of FoxD5b expression by PV.1 may be mediated by a Hox gene.

\section{DISCUSSION}

BMP signaling is involved in various cell fate specification in of vertebrate embryogenesis (Dale and Wardle, 1999; Dosch et al., 1997; Glinka et al., 1997; Hawley et al., 1995; Wilson and Hemmati-Brivanlou, 1995). During the early development of Xenopus embryos, BMP signaling gradients modulate dorso-ventral patterning and negatively regulate neural induction (Dosch et al., 1997). The Spemann's organizer, which is located in the dorsal mesoderm, produces BMP antagonists and reduces BMP signaling (Hawley et al., 1995). The suppression of BMP signaling induces neural fate in the ectoderm (Xu et al., 1995). BMP signaling induces numerous target genes including MSX1, Vents and GATAs, which can inhibit neural gene expression (Chung and Chung, 1999; Gawantka et al., 1995; Hwang et al., 2003; Rogers et al., 2008; Shibata et al., 1998; Suzuki et al., 1997). However, the mechanism by which BMP signaling suppresses neural induction is not fully understood.

Our previous research has shown that the FoxD5a and FoxD5b promoters have two highly conserved regions. The suppression of BMP signaling induces FoxD5b expression through AP-1C-Jun/FosB. Interestingly, the AP-1 binding site is a conserved region. Furthermore, we also found that the over- expression of BMP4 strongly reduced the activity of the FoxD5b promoter in the entire embryo (data not shown). These data suggest that the FoxD5b promoter has a negative response element that is regulated by BMP signaling.

PV.1 has roles in ventralization and inhibition of neural induction that are mediated by BMP signaling via its c-terminal repressor domain (Hwang et al., 2003). Here, we investigated how PV.1 regulates the expression of FoxD5b, which is an early neural gene. PV.1 and FoxD5b are highly expressed in the blastula stage. Over-expression of PV.1 reduced FoxD5b expression and the promoter activity of FoxD5b. These results are consistent with the phenotypical changes of the whole embryos. Additionally, we analyzed whether PV.1 directly suppressed FoxD5b expression using a cyclohexamide treatment. As shown in Figs. 2A and 2B, PV.1 directly suppressed FoxD5b and Zic3 expression. The luciferase assay with serially truncated FoxD5b promoters demonstrated that the PV.1 response element is located between -301 and -186 . However, no putative binding site was found in this region. A ChIP assay also revealed that PV.1 did not interact at this region (data not shown). Furthermore, we analyzed the expression of the luciferase gene for the FoxD5b promoter in cyclohexamide-treated animal cap explants (Fig. 4A). These results suggest that our FoxD5b promoter did not contain a direct response element for PV.1. Sequence analysis of theFoxD5b promoter using bioinformatics revealed that a putative Hox binding site exists in the -301 region. Hox family genes have been known to regulate dorsoventral and anterioposterior patterning via the mediation 
A

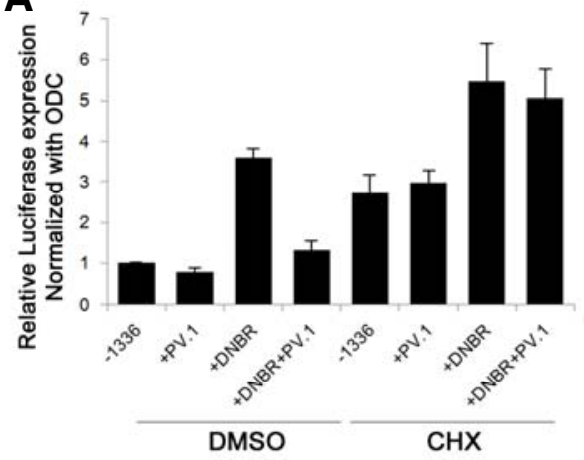

C

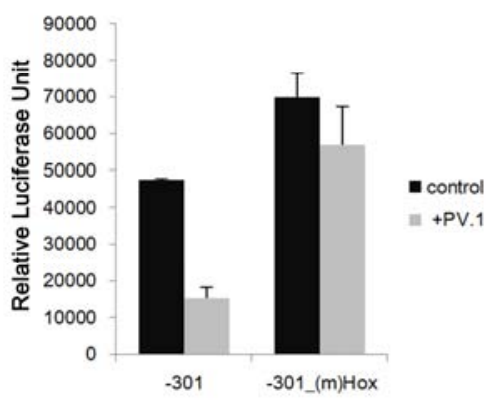

B

ATGCATAAGA $\Rightarrow$ ATGCGCGAGA

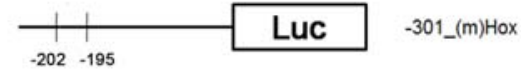

D

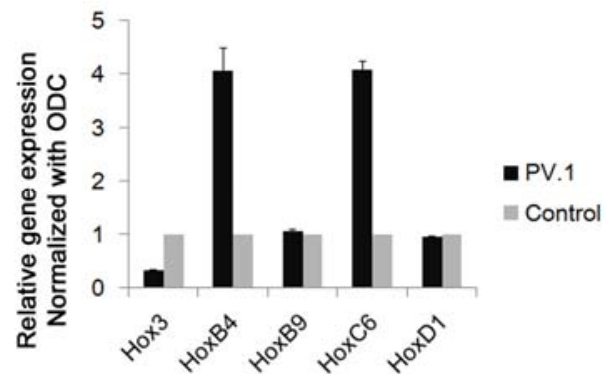

Fig. 4. PV.1 regulates FoxD5b expression indirectly via the putative Hox binding site. (A) Embryos were co-injected 1336 construct with RNAs (PV.1, $500 \mathrm{pg}$ and DNBR, 1 ng) at the one-cell stage as indicated. Embryos were harvested at stage 10 for analyses of the relative luciferase gene expressions using RTPCR. (B) Schematic representation of -301_(m)Hox construct (C) The -301 or -301_(m) Hox constructs were either injected alone or co-injected with PV.1 at the one-cell stage. Luciferase activity was measured at stage 10. (D) RNAs of PV.1 (500 pg) were injected at the one-cell stage. Animal caps were dissected at stage 8 and incubated until stage 10. RT-PCR was performed for the analysis of the expression of the indicated genes. The data are shown as the means \pm the S.D.s of

the values of at least three independent experiments. Differences were considered significant at $P<0.05$.

of BMP signaling during the early development of vertebrate embryos. Additionally, our results showed that the over-expression of PV.1 increased the expression of several Hox genes (Fig. 4D). Furthermore, a point mutation of the putative Hox binding in the -301 construct demonstrated that the putative Hox binding site is involved with FoxD5b expression, which is regulated by PV.1. Taken together, we suggest that some Hox genes cooperatively regulate FoxD5b expression with PV.1. However, which Hox genes participate in this process is still unclear.

Although the over-expression of BMP downstream target genes, including MSX1, GATA and Vents, is sufficient for the suppression of neural fate, no data exists that suggests that the knock-down of these genes induces neural tissue. In this study, we also examined whether the knock down of PV.1 induced neural induction. The micro-injection of PV.1 morpholino oligos slightly increased the expression of Zic3 and FoxD5b at stage 10 , but these treatments did not induce any neural marker at stage 24. This result indicates that the knock-down of PV.1 alone was not sufficient to induce neurogenesis. In other words, PV.1 and some BMP down-stream target genes cooperatively suppress the neural gene expression.

\section{ACKNOWLEDGMENTS}

This research was supported by the Basic Science Research Program through the National Research Foundation of Korea (NRF), funded by the Ministry of Education, Science and Technology (NRF-2013R1A1A2008541) and Hallym University Research Fund (HRF-G-2012-4).

\section{REFERENCES}

Ault, K.T., Dirksen, M.L., and Jamrich, M. (1996). A novel homeobox gene PV.1 mediates induction of ventral mesoderm in Xenopus embryos. Proc. Natl. Acad. Sci. USA 93, 6415-6420.

Ault, K.T., Xu, R.H., Kung, H.F., and Jamrich, M. (1997). The homeobox gene PV.1 mediates specification of the prospective neural ectoderm in Xenopus embryos. Dev. Biol. 192, 162-171.

Chung, H.G., and Chung, H.M. (1999). Neuregulin induces the expression of mesodermal genes in the ectoderm of Xenopus laevis. Mol. Cells 9, 497-503.

Dale, L., and Jones, C.M. (1999). BMP signalling in early Xenopus development. Bioessays 21, 751-760.

Dale, L., and Wardle, F.C. (1999). A gradient of BMP activity specifies dorsal-ventral fates in early Xenopus embryos. Semin. Cell Dev. Biol. 10, 319-326.

Dosch, R., Gawantka, V., Delius, H., Blumenstock, C., and Niehrs, C. (1997). Bmp-4 acts as a morphogen in dorsoventral mesoderm patterning in Xenopus. Development (Cambridge, England) $124,2325-2334$.

Fetka, I., Doederlein, G., and Bouwmeester, T. (2000). Neuroectodermal specification and regionalization of the Spemann organizer in Xenopus. Mech. Dev. 93, 49-58.

Friedle, H., and Knochel, W. (2002). Cooperative interaction of Xvent-2 and GATA-2 in the activation of the ventral homeobox gene Xvent-1B. J. Biol. Chem. 277, 23872-23881.

Gawantka, V., Delius, H., Hirschfeld, K., Blumenstock, C., and Niehrs, C. (1995). Antagonizing the Spemann organizer: role of the homeobox gene Xvent-1. EMBO J. 14, 6268-6279.

Glinka, A., Wu, W., Onichtchouk, D., Blumenstock, C., and Niehrs, C. (1997). Head induction by simultaneous repression of Bmp and Wnt signalling in Xenopus. Nature 389, 517-519.

Hawley, S.H., Wunnenberg-Stapleton, K., Hashimoto, C., Laurent, M.N., Watabe, T., Blumberg, B.W., and Cho, K.W. (1995). Disruption of BMP signals in embryonic Xenopus ectoderm leads to direct neural induction. Genes Dev. 9, 2923-2935.

Hwang, Y.S., Seo, J.J., Cha, S.W., Lee, H.S., Lee, S.Y., Roh, D.H., Kung Hf, H.F., Kim, J., and Ja Park, M. (2002). Antimorphic 
PV.1 causes secondary axis by inducing ectopic organizer. Biochem. Biophys. Res. Commun. 292, 1081-1086.

Hwang, Y.S., Lee, H.S., Roh, D.H., Cha, S., Lee, S.Y., Seo, J.J., Kim, J., and Park, M.J. (2003). Active repression of organizer genes by C-terminal domain of PV.1. Biochem. Biophys. Res. Commun. 308, 79-86.

Jackson, B.C., Carpenter, C., Nebert, D.W., and Vasiliou, V. (2010). Update of human and mouse forkhead box (FOX) gene families. Hum. Genomics 4, 345-352.

Katoh, M., and Katoh, M. (2004). Human FOX gene family (Review). Int. J. Oncol. 25, 1495-1500.

Katoh, M., Igarashi, M., Fukuda, H., Nakagama, H., and Katoh, M. (2012). Cancer genetics and genomics of human FOX family genes. Cancer Lett. 328, 198-206.

Kuroda, H., Fuentealba, L., Ikeda, A., Reversade, B., and De Robertis, E.M. (2005). Default neural induction: neuralization of dissociated Xenopus cells is mediated by Ras/MAPK activation. Genes Dev. 19, 1022-1027.

Lee, H.C., Tseng, W.A., Lo, F.Y., Liu, T.M., and Tsai, H.J. (2009). FoxD5 mediates anterior-posterior polarity through upstream modulator Fgf signaling during zebrafish somitogenesis. Dev. Biol. 336, 232-245.

Lee, H.S., Lee, S.Y., Lee, H., Hwang, Y.S., Cha, S.W., Park, S., Lee, J.Y., Park, J.B., Kim, S., Park, M.J., et al. (2011). Direct response elements of BMP within the PV.1A promoter are essential for its transcriptional regulation during early Xenopus development. PLoS One 6, e22621.

Moore, K.B., Mood, K., Daar, I.O., and Moody, S.A. (2004). Morphogenetic movements underlying eye field formation require interactions between the FGF and ephrinB1 signaling pathways. Dev. Cell 6, 55-67.

Nieuwkoop, P.D., and Faber, J. (1967). Normal table of Xenopus laevis, Vol. 2nd ed. (Northe Holland, Amsterdam).

Pohl, B.S., and Knochel, W. (2005). Of Fox and Frogs: Fox (fork head/winged helix) transcription factors in Xenopus development. Gene 344, 21-32.

Rogers, C.D., Archer, T.C., Cunningham, D.D., Grammer, T.C., and Casey, E.M. (2008). Sox3 expression is maintained by FGF signaling and restricted to the neural plate by Vent proteins in the Xenopus embryo. Dev. Biol. 313, 307-319.
Rogers, C.D., Moody, S.A., and Casey, E.S. (2009). Neural induction and factors that stabilize a neural fate. Birth Defects Res. C Embryo Today 87, 249-262.

Shibata, K., Ishimura, A., and Maeno, M. (1998). GATA-1 inhibits the formation of notochord and neural tissue in Xenopus embryo. Biochem. Biophys. Res. Commun. 252, 241-248.

Smith, J.C., and Slack, J.M. (1983). Dorsalization and neural induction: properties of the organizer in Xenopus laevis. J. Embryol. Exp. Morphol. 78, 299-317.

Sullivan, S.A., Akers, L., and Moody, S.A. (2001). foxD5a, a Xenopus winged helix gene, maintains an immature neural ectoderm via transcriptional repression that is dependent on the Cterminal domain. Dev. Biol. 232, 439-457.

Suzuki, A., Ueno, N., and Hemmati-Brivanlou, A. (1997). Xenopus msx1 mediates epidermal induction and neural inhibition by BMP4. Development (Cambridge, England) 124, 3037-3044.

Taylor, J.J., Wang, T., and Kroll, K.L. (2006). Tcf- and Vent-binding sites regulate neural-specific geminin expression in the gastrula embryo. Dev. Biol. 289, 494-506.

Wacker, S.A., McNulty, C.L., and Durston, A.J. (2004). The initiation of Hox gene expression in Xenopus laevis is controlled by Brachyury and BMP-4. Dev. Biol. 266, 123-137.

Wilson, P.A., and Hemmati-Brivanlou, A. (1995). Induction of epidermis and inhibition of neural fate by Bmp-4. Nature 376, 331333

Xu, R.H., Kim, J., Taira, M., Zhan, S., Sredni, D., and Kung, H.F. (1995). A dominant negative bone morphogenetic protein 4 receptor causes neuralization in Xenopus ectoderm. Biochem. Biophys. Res. Commun. 212, 212-219.

Yan, B., Neilson, K.M., and Moody, S.A. (2009a). foxD5 plays a critical upstream role in regulating neural ectodermal fate and the onset of neural differentiation. Dev. Biol. 329, 80-95.

Yan, B., Neilson, K.M., and Moody, S.A. (2009b). Notch signaling downstream of foxD5 promotes neural ectodermal transcription factors that inhibit neural differentiation. Dev. Dyn. 238, 13581365.

Yu, J.K., Holland, N.D., and Holland, L.Z. (2002). An amphioxus winged helix/forkhead gene, AmphiFoxD: insights into vertebrate neural crest evolution. Dev. Dyn. 225, 289-297. 